\title{
BMJ Open Early warning scores for detecting deterioration in adult hospital patients: a systematic review protocol
}

\author{
Stephen Gerry, ${ }^{1}$ Jacqueline Birks, ${ }^{1}$ Timothy Bonnici, ${ }^{2}$ Peter J Watkinson, ${ }^{3}$ \\ Shona Kirtley, ${ }^{4}$ Gary S Collins ${ }^{1}$
}

To cite: Gerry S, Birks J, Bonnici T, et al. Early warning scores for detecting deterioration in adult hospital patients: a systematic review protocol. BMJ Open 2017;7:e019268. doi:10.1136/ bmjopen-2017-019268

- Prepublication history and additional material for this paper are available online. To view these files, please visit the journal online (http://dx.doi. org/10.1136/bmjopen-2017019268).

Received 21 August 2017 Revised 28 September 2017 Accepted 2 October 2017

\section{CrossMark}

${ }^{1}$ Centre for Statistics in Medicine, Nuffield Department of Orthopaedics, Rheumatology \& Musculoskeletal Sciences, University of Oxford, Oxford, UK ${ }^{2}$ Nuffield Department of Medicine, University of Oxford, Oxford, UK

${ }^{3}$ Kadoorie Centre for Critical Care Research and Education, Nuffield Department of Clinical Neurosciences, University of Oxford, Oxford, UK ${ }^{4}$ UK EQUATOR Centre, Centre for Statistics in Medicine, Nuffield Department of Orthopaedics, Rheumatology \& Musculoskeletal Sciences, University of Oxford, 0xford, UK

Correspondence to

Stephen Gerry;

stephen.gerry@csm.ox.ac.uk

\section{ABSTRACT}

Introduction Early warning scores (EWSs) are used extensively to identify patients at risk of deterioration in hospital. Previous systematic reviews suggest that studies which develop EWSs suffer methodological shortcomings and consequently may fail to perform well. The reviews have also identified that few validation studies exist to test whether the scores work in other settings. We will aim to systematically review papers describing the development or validation of EWSs, focusing on methodology, generalisability and reporting.

Methods We will identify studies that describe the development or validation of EWSs for adult hospital inpatients. Each study will be assessed for risk of bias using the Prediction model Risk of Bias ASsessment Tool (PROBAST). Two reviewers will independently extract information. A narrative synthesis and descriptive statistics will be used to answer the main aims of the study which are to assess and critically appraise the methodological quality of the EWS, to describe the predictors included in the EWSs and to describe the reported performance of EWSs in external validation.

Ethics and dissemination This systematic review will only investigate published studies and therefore will not directly involve patient data. The review will help to establish whether EWSs are fit for purpose and make recommendations to improve the quality of future research in this area.

PROSPERO registration number CRD42017053324.

\section{BACKGROUND}

Towards the end of the 20th century, accumulating evidence suggested that people in hospital wards were dying and suffering harm unnecessarily. ${ }^{1-3}$ Multiple studies have demonstrated that cardiac arrest or death is commonly preceded by several hours of deranged physiology. ${ }^{4-6}$ Recommendations were made to put systems in place to use this information to identify and respond to previously unrecognised deterioration in patients. ${ }^{7}$ In response, the first early warning score (EWS) was published in 1997.8

EWSs are simple tools to reduce unnecessary harm in hospitals. These clinical

\section{Strengths and limitations of this study}

The first systematic review in a decade to include all published early warning scores (EWSs).

- The first systematic review to include EWS validation studies.

- The review will assess the methodology and generalisability of studies to identify the best current EWSs and make recommendations for future development and validation studies.

- The review will be limited to examining published EWSs. Many other scores may be in clinical use, but not published.

prediction models use patients' measured vital signs to monitor their health during their hospital stay and identify their likelihood of deteriorating, characterised as death or admission to intensive care unit (ICU), for example. Should a patient show signs of deteriorating, the EWS triggers a warning so that care can be escalated. EWSs, which are also commonly referred to as track-and-trigger scores, are often implemented as part of an 'early warning system' or 'EWS system'. These are computer systems which record vital signs, automatically or manually and then implement the EWS algorithm to indicate a patient's risk of deterioration. The interest of this review lies in the underlying scoring systems/algorithms themselves and not the systems in which they are implemented.

There are now many EWSs available. ${ }^{9-11}$ They are routinely used in several countries, including the Netherlands, USA and Australia and their use in UK hospitals is mandated as a standard of care by the National Institute For Health and Clinical Excellence (NICE).$^{12}$ Based on the Hospital Episode Statistics, ${ }^{13}$ we estimate that EWSs are used more than 120 million times per year in the NHS in England alone, a 
conservative estimate that probably well underestimates the true total. ${ }^{\text {i }}$

EWSs have been derived using a variety of approaches. Some have been developed using statistical methods for clinical prediction, by linking observations (eg, vital signs) to outcomes (eg, death, ICU admission) through regression models. Others have been based on clinical consensus without statistical modelling. Although there is now an abundance of clinical prediction models in many fields of medicine and healthcare, in practice many of these models are scarcely used. ${ }^{14}{ }^{15}$ Systematic reviews of clinical prediction models in other clinical areas have all concluded that many are poorly developed $^{15-17}$ and that they are rarely and inappropriately evaluated $^{1819}$ (often referred to as validation), that is, tested in different settings to which they were developed. There is no common agreement on which of the dozens of EWSs available performs best. Most problematically, recent evidence suggests that EWSs have not solved the problem they were designed for: unrecognised deterioration of patients in hospitals remains a major issue. ${ }^{20}$

The aim of this systematic review is to critically appraise papers describing the development and validation of EWSs for adult hospital inpatients, with a particular focus on methodology, reporting and generalisability, in order to identify high quality EWSs and provide guidance regarding the methods to develop and validate future EWSs.

\section{Existing systematic reviews}

Four systematic reviews of studies which develop or validate EWSs have been published. ${ }^{9-11}$ Those by Gao et a $\ell^{9}$ and GB Smith et $a l^{10}$ were published almost a decade ago, while MEB Smith et $a l^{11}$ used narrow inclusion criteria and did not include all available EWSs, ${ }^{11}$ and the review by Kyriacos $e t a l^{21}$ was a more general overview of the literature. Several new EWSs have been published since.

The main aims of the reviews were to describe the development of EWSs, assess their predictive performance and assess any impact studies that evaluate the effect of implementing EWSs in clinical practice. Other reviews, such as those by Alam et $a l^{22}$ and McGaughey et $a l^{23}$ looked at impact studies, but we do not plan to include these in our review.

Many of the reviewed scores included similar predictors and applied similar weights to those predictors. Nearly all of the scores included pulse rate, breathing rate, systolic blood pressure and temperature. The reviews also found some indication that scores that included age performed better. ${ }^{10}$ In contrast to studies developing EWSs, validation studies that evaluated the performance of EWSs were relatively uncommon.

${ }^{\mathrm{i}}(\sim 12$ million non-day-cases per year $*$ mean length of stay 5 days $* 2$ observations per day).
The use of poor methods to develop EWSs could mean that the scores are unreliable and fail to accurately predict risk. Gao et al ${ }^{9}$ and MEB Smith $e a^{11}$ subjectively reported that they found many of the primary studies to be of low quality, used suboptimal methods and were at high risk of bias. ${ }^{911}$ However, none of the reviews made a detailed and structured evaluation of the approaches used to develop EWSs, following recommended methodological considerations in the field of clinical prediction models. ${ }^{24-28}$

After a prediction model (ie, an EWS) has been developed, its predictive accuracy should be evaluated in the same population used to derive it, a process called internal validation. The two widely recommended characteristics that describe the performance of a prediction model are discrimination (eg, the c-index and AUROC) and calibration. ${ }^{24}$ Discrimination reflects a prediction model's ability to differentiate between those who develop an outcome (ie, death) and those who do not. A model should predict higher risks for those who develop the outcome. Calibration reflects the level of agreement between observed outcomes and the model's predictions.

Both discrimination and calibration must be assessed and reported to judge a model's accuracy. ${ }^{24}$ However, as in many other clinical areas, studies evaluating EWSs have tended to give more prominence to discrimination and have rarely assessed model calibration. Two of the reviews investigated how primary EWS studies report predictive performance, with conflicting conclusions. Gao et $a \ell^{\dagger}$ found unacceptance predictive performance, ${ }^{9}$ whereas MEB Smith et $a l^{11}$ found good predictive performance. This difference in result may reflect differences in the included studies and how the authors assessed model performance.

Internal validation provides insights into model performance in the same population used to derive the model. In contrast, external validation assesses the model's performance in a different population from that used to derive it. External validation assesses model discrimination and calibration to determine whether the model performs satisfactorily in data other than that it was developed with, which is called generalisability. ${ }^{29}$ Although the four reviews did not have a specific focus on external validation studies, they all highlighted a lack of external validation studies of EWSs. GB Smith et $a l^{10}$ did not investigate validation studies, but performed their own external validation as part of their review by evaluating the identified models using their own data. They found that none of the scores showed good enough performance. ${ }^{10}$

\section{Research aims}

In this systematic review, we aim to identify all existing published EWSs for adult hospital inpatients and:

1. Describe and critically appraise the methods that have been used to develop and validate (where appropriate) the scores. We will take a wide-ranging approach and will cover statistical aspects, such as how missing data are accounted for and how continuous 
predictors are used. We will also investigate aspects of generalisability, such as details of the populations used to develop the models.

2. Describe which predictors are included in the scores and how they are weighted.

3. Report which EWSs have undergone external validation and, if so, how well they performed.

\section{METHODS}

Our systematic review protocol was registered with the International Prospective Register of Systematic Reviews (PROSPERO) on 12 July 2017 (registration number CRD42017053324). Our systematic review will be carried out and reported in accordance with two published guidelines: the Critical Appraisal and Data Extraction for Systematic Reviews of Prediction Modelling Studies (CHARMS) checklist ${ }^{30}$ and the Preferred Reporting Items for Systematic Reviews and Meta-Analyses (PRISMA) checklist. ${ }^{31}$

\section{Selection criteria}

We will include studies that satisfy all of the following criteria:

1. The study describes the development or validation of one or more EWSs, defined as a score used to identify hospitalised patients at risk of clinical deterioration.

2. The EWS studied combines information from at least two predictor variables to produce a summary risk estimate.

3. Validation studies will only be included where the corresponding development articles are available.

We will exclude papers where any of the following apply:

1. The score was developed for use in a subset of patients with a specific disease or group of diseases.

2. The score was developed for use with children (aged under 16 years) or pregnant women.

3. The score is intended for outpatient use.

4. The score is intended for use in the ICU.

5. Reviews, letters, personal correspondence and abstracts.

\section{Search strategy}

Studies will be identified by searching the medical literature using Medline (OVID), CINAHL (EbscoHost) and Embase (OVID) to identify primary articles reporting on the development and/or validation of EWSs. We will use a combination of relevant controlled vocabulary terms for each database (eg, MeSH, Emtree) and free-text search terms. No date or language restrictions will be applied. Citation lists of previous systematic reviews and included studies will be searched to identify any studies missed by the search. We will also conduct a Google Scholar search to identify any other eligible studies. Online supplementary appendix A shows a draft search strategy.

\section{Study selection}

Two reviewers will independently screen all titles and abstracts using prespecified screening criteria. The full text of any relevant articles will then be independently assessed by two reviewers. Disagreements will be resolved by discussion and, if necessary, referral to a third reviewer. The study selection process will be reported using a PRISMA flow diagram. ${ }^{31}$

\section{Data extraction}

Data will be independently extracted by two reviewers using a standardised and piloted data extraction form. The form will be administered using the Research Electronic Data Capture (REDCap) electronic data capture tool. ${ }^{32}$ Disagreements will be resolved by discussion and, if necessary, by referral to a third reviewer. We will choose items for extraction based on the CHARMS checklist, ${ }^{30}$ supplemented by subject-specific questions and methodological guidance. Items for extraction will include:

- Study characteristics (development and validation) (eg, country, year).

- Study design (development and validation) (eg, prospective, case control, cohort, clinical consensus).

- Patient characteristics (development and validation) (eg, hospital ward, age, sex).

- Predicted outcome (development and validation) (eg, survival at 24 hours, ICU admission at 24 hours).

- Model development (development) (eg, sample size, type of model, handling of continuous variables, selection of variables, missing data, method of internal validation).

- Model presentation (development) (eg, full regression model, simplified model, risk groups).

- Assessment of performance (development and validation) (eg, measures of discrimination, measures of calibration).

\section{Assessment of bias}

Each article will be independently assessed by two reviewers using the Prediction model Risk of Bias ASsessment Tool (PROBAST), which was recently developed by the Cochrane Prognosis Methods Group to assess the quality and risk of bias for prediction models (due to be submitted shortly; Wolff R, Whiting, Mallett S et al. (including author GSC), personal communication). PROBAST consists of 23 signalling questions within four domains (participant selection, predictors, outcome and analysis).

\section{Evidence synthesis}

We will summarise the results using descriptive statistics, graphical plots and a narrative synthesis. We do not plan to perform a quantitative synthesis of the scores or their predictive performance. However, if we identify multiple studies that evaluate the same EWS and report common performance measures, we will summarise their performance using a random-effects meta-analysis. ${ }^{33}$ The PROBAST evaluation will be used to determine the models' risk of bias, including whether the EWSs are likely to work as intended for the hospital population of interest. The models will be classed as low, high or unclear risk of bias. 


\section{DISCUSSION}

Although EWSs are extensively used in clinical practice, the methodology behind them remains questionable. Although not formally assessed, previous systematic reviews of EWSs have indicated that many studies suffer from a lack of quality and that few EWSs have been satisfactorily validated. ${ }^{9-11}$ These aspects are crucial for developing a prediction model that can confidently be rolled out into clinical practice. This systematic review will bridge this important gap by examining methodological quality and external validation in detail. This systematic review is timely, as it is now nearly a decade since the last comprehensive review of EWSs, which have only existed for 20 years.

EWSs have historically been implemented as part of traditional paper observation charts. The requirement for scores to be calculated manually necessitated the use of simple scoring algorithms. Storage of data on paper has been a barrier to collection of large datasets for score derivation and validation. Digital systems are increasingly being used to record vital signs and calculate EWSs, ${ }^{34}$ offering the opportunity to be more rigorous and innovative in the development and implementation of new EWSs. The adoption of digital vital signs charting offers an opportunity to transition away from poor quality EWSs. Our review will provide the evidence for creators of digital systems to identify which EWSs should be prioritised for implementation.

Contributors SG, JB, TB, PJW and GSC conceived the study. SG developed the study protocol and will implement the systematic review under the supervision of GSC. SG will provide the study's statistical analysis plan and will analyse the data. $S G$ and SK will perform the study search and SG will screen and extract the data. JB, TB, PJW and GSC will review the work. SG wrote the first protocol manuscript draft and all authors gave input into and approved the final draft of the protocol.

Funding SG is funded by an NIHR Doctoral Fellowship (DRF-2016-09-073). JB, TB, PJW and GSC are supported by the NIHR Biomedical Research Centre, Oxford. The funders have not played any role in the development of this protocol.

Competing interests None declared.

Provenance and peer review Not commissioned; externally peer reviewed.

Data sharing statement All unpublished data will be made available on request. The first author, SG, should be contacted with requests for unpublished data.

Open Access This is an Open Access article distributed in accordance with the terms of the Creative Commons Attribution (CC BY 4.0) license, which permits others to distribute, remix, adapt and build upon this work, for commercial use, provided the original work is properly cited. See: http://creativecommons.org/ licenses/by/4.0/

(C) Article author(s) (or their employer(s) unless otherwise stated in the text of the article) 2017. All rights reserved. No commercial use is permitted unless otherwise expressly granted.

\section{REFERENCES}

1. Brennan TA, Leape LL, Laird NM, et al. Incidence of adverse events and negligence in hospitalized patients. N Engl J Med 1991;324:370-6.

2. Institute of Medicine Committee on Quality of Health Care in A. In: Kohn LT, Corrigan JM, Donaldson MS, eds. To Err is human: building a safer health system. Washington DC: National Academies Press (US), 2000

3. Vincent C, Neale G, Woloshynowych M. Adverse events in British hospitals: preliminary retrospective record review. BMJ 2001;322:517-9.
4. Hillman KM, Bristow PJ, Chey T, et al. Duration of life-threatening antecedents prior to intensive care admission. Intensive Care Med 2002;28:1629-34.

5. Kause J, Smith G, Prytherch D, et al. A comparison of antecedents to cardiac arrests, deaths and emergency intensive care admissions in Australia and New Zealand, and the United Kingdom--the ACADEMIA study. Resuscitation 2004;62:275-82.

6. Hogan H, Healey F, Neale G, et al. Preventable deaths due to problems in care in English acute hospitals: a retrospective case record review study. BMJ Qual Saf 2012;21:737-45.

7. McQuillan P, Pilkington S, Allan A, et al. Confidential inquiry into quality of care before admission to intensive care. BMJ 1998;316:1853-8.

8. Morgan RJM, Williams F, Wright MM. An early warning scoring system for detecting developing critical illness. Clin Intensive Care 1997:8:100.

9. Gao H, McDonnell A, Harrison DA, et al. Systematic review and evaluation of physiological track and trigger warning systems for identifying at-risk patients on the ward. Intensive Care Med 2007;33:667-79.

10. Smith GB, Prytherch DR, Schmidt PE, et al. Review and performance evaluation of aggregate weighted 'track and trigger' systems. Resuscitation 2008;77:170-9.

11. Smith ME, Chiovaro JC, O'Neil M, et al. Early warning system scores for clinical deterioration in hospitalized patients: a systematic review. Ann Am Thorac Soc 2014;11:1454-65.

12. NICE. Acutely ill adults in hospital: recognising and responding to deterioration (NICE guideline CG50), 2007.

13. Centre HSCl. Hospital Episode Statistics. Admitted Patient Care, England - 2014-15, 2015.

14. Damen JA, Hooft L, Schuit E, et al. Prediction models for cardiovascular disease risk in the general population: systematic review. BMJ 2016;353:i2416.

15. Kleinrouweler CE, Cheong-See FM, Collins GS, et al. Prognostic models in obstetrics: available, but far from applicable. Am J Obstet Gynecol 2016;214:79-90.

16. Bouwmeester W, Zuithoff NP, Mallett S, et al. Reporting and methods in clinical prediction research: a systematic review. PLoS Med 2012;9:1-12.

17. Collins GS, Mallett S, Omar O, et al. Developing risk prediction models for type 2 diabetes: a systematic review of methodology and reporting. BMC Med 2011;9:103.

18. Collins GS, de Groot JA, Dutton S, et al. External validation of multivariable prediction models: a systematic review of methodological conduct and reporting. BMC Med Res Methodol 2014; $14: 40$.

19. Mallett S, Royston P, Waters R, et al. Reporting performance of prognostic models in cancer: a review. BMC Med 2010;8:21.

20. NCEPOD. Time to Intervene? A review of patients who underwent cardiopulmonary rescuscitation as a result of an in-hospital cardiorespiratory arrest, 2012.

21. Kyriacos U, Jelsma J, Jordan S. Monitoring vital signs using early warning scoring systems: a review of the literature. J Nurs Manag 2011:19:311-30.

22. Alam N, Hobbelink EL, van Tienhoven AJ, et al. The impact of the use of the Early Warning Score (EWS) on patient outcomes: a systematic review. Resuscitation 2014;85:587-94.

23. McGaughey J, Alderdice F, Fowler R, et al. Outreach and Early Warning Systems (EWS) for the prevention of intensive care admission and death of critically ill adult patients on general hospital wards. Cochrane Database Syst Rev 2007;3:CD005529.

24. Collins GS, Reitsma JB, Altman DG, et al. Transparent Reporting of a multivariable prediction model for Individual Prognosis or Diagnosis (TRIPOD): the TRIPOD statement. Ann Intern Med 2015;162:55-63.

25. Moons KG, Altman DG, Reitsma JB, et al. Transparent Reporting of a multivariable prediction model for Individual Prognosis or Diagnosis (TRIPOD): explanation and elaboration. Ann Intern Med 2015;162:W1-73.

26. Moons KG, Kengne AP, Woodward M, et al. Risk prediction models: I. Development, internal validation, and assessing the incremental value of a new (bio)marker. Heart 2012;98:683-90.

27. Steyerberg EW, Moons KG, van der Windt DA, et al. PROGRESS Group. Prognosis Research Strategy (PROGRESS) 3: prognostic model research. PLoS Med 2013;10:e1001381.

28. Steyerberg EW, Vergouwe Y. Towards better clinical prediction models: seven steps for development and an ABCD for validation. Eur Heart J 2014;35:1925-31.

29. Justice AC, Covinsky KE, Berlin JA. Assessing the generalizability of prognostic information. Ann Intern Med 1999;130:515-24. 
30. Moons KG, de Groot JA, Bouwmeester W, et al. Critical appraisal and data extraction for systematic reviews of prediction modelling studies: the CHARMS checklist. PLoS Med 2014;11:e1001744.

31. Moher D, Liberati A, Tetzlaff J, et al. Preferred reporting items for systematic reviews and meta-analyses: the PRISMA statement. PLoS Med 2009;6:e1000097.

32. Harris PA, Taylor R, Thielke R, et al. Research electronic data capture (REDCap)--a metadata-driven methodology and workflow process for providing translational research informatics support. J Biomed Inform 2009;42:377-81.

33. Debray TP, Damen JA, Snell KI, et al. A guide to systematic review and meta-analysis of prediction model performance. BMJ 2017;356:i6460.

34. Wong D, Bonnici T, Knight J, et al. SEND: a system for electronic notification and documentation of vital sign observations. BMC Med Inform Decis Mak 2015;15:68. 